Int Surg 2012;97:245-248

\title{
Spontaneous Intraperitoneal Rupture of a Hepatic Hydatid Cyst
}

\author{
Zulfu Arikanoglu ${ }^{1}$, Fatih Taskesen ${ }^{1}$, İbrahim Aliosmanoğlu ${ }^{1}$, Mesut Gul ${ }^{1}$, \\ Mehmet Guli Cetincakmak², Akin Onder ${ }^{1}$, Murat Kapan ${ }^{1}$ \\ ${ }^{1}$ Department of Surgery and ${ }^{2}$ Department of Radiology, Dicle University Faculty of Medicine, \\ Diyarbakir, Turkey
}

\begin{abstract}
Hydatid cysts, which are endemic to certain areas, typically are found in the liver. Spontaneous intraperitoneal rupture, which can be life threatening, is rare. This article presents a case of spontaneous rupture of a hydatid cyst in a 69-year-old woman who was admitted to the emergency department. The patient had no history of trauma. Abdominal ultrasonography and computed tomography suggested rupture of a hydatid cyst. The patient underwent a partial cystectomy, and the cystic area was washed with hypertonic saline and the peritoneal cavity was washed with isotonic saline and drained. Postoperatively, the patient was treated with albendazole for 3 months. No additional pathology was observed at the 3-, 6-, and 9-month follow-ups. Although rare, a ruptured hydatid cyst should be considered in the differential diagnosis of the acute abdomen in a patient residing in an endemic area.
\end{abstract}

Key words: Liver - Hydatid cyst - Spontaneous rupture

$\mathrm{H}$ ydatid disease is a parasitic infection caused by Echinococcus granulosus. Although the parasite most frequently settles in the liver and lungs, it may be encountered in any part of the body. ${ }^{1}$ Experienced surgeons are aware that a hydatid cyst may rupture, either spontaneously or after trauma or surgery. Although hepatic hydatid cysts rupture most commonly into the biliary tree, they may also rupture into the blood vessels, bronchi, or peritoneal cavity. Spontaneous intraperitoneal rupture of a cyst, albeit rare $(1 \%-8 \%)$, is life threatening. ${ }^{2}$ Patients with hydatid cyst rupture into the peritoneal cavity are often admitted to the emergency department with an acute abdomen. The diagnosis is established by ultrasonography (USG) and computed tomography (CT). ${ }^{3}$

This article presents a rare case of spontaneous rupture of a hydatid cyst in a 69-year-old woman admitted to the Dicle University Hospital Emergency Department complaining of pain in the abdomen and shortness of breath. 


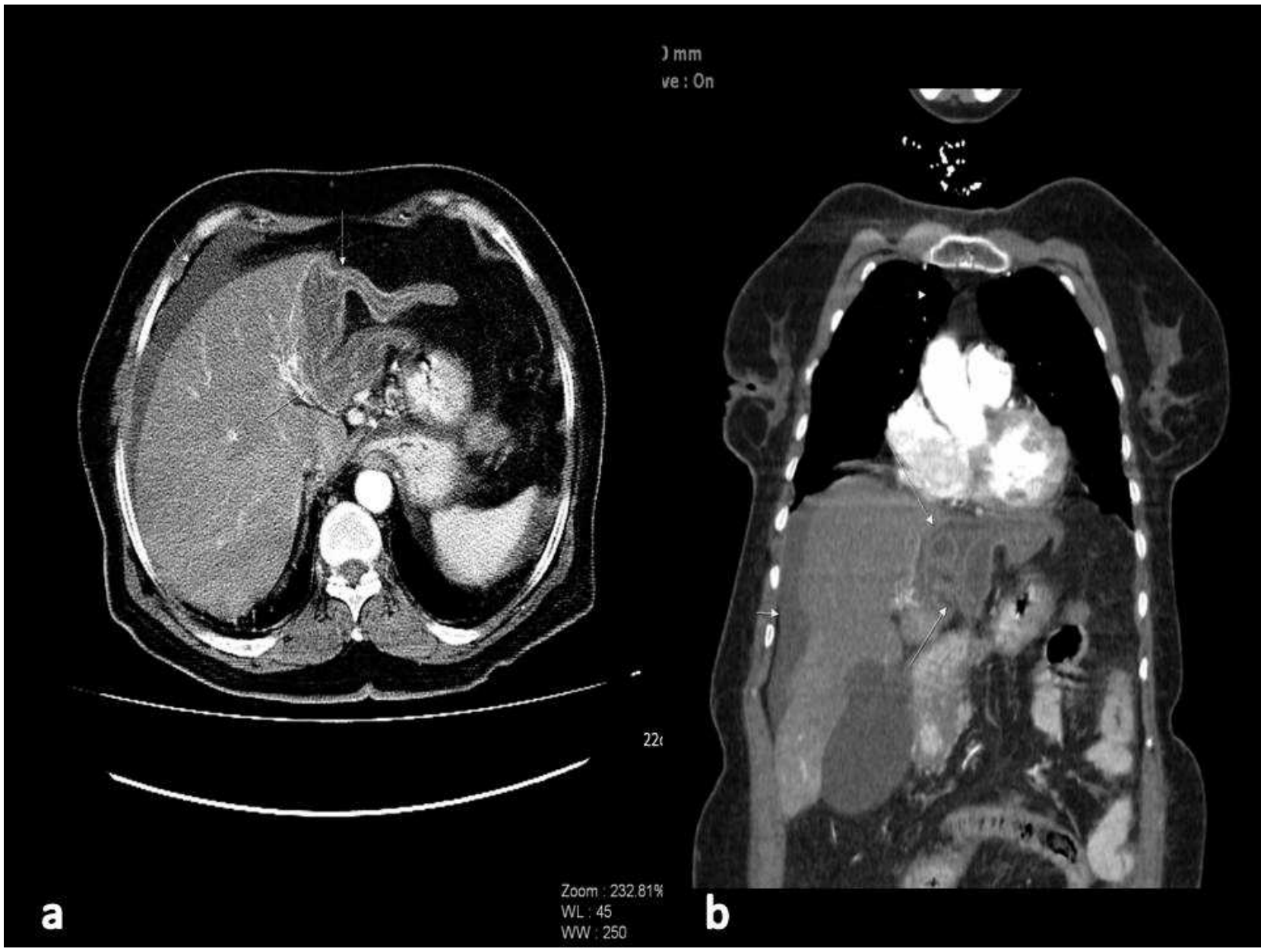

Fig. 1 Post-contrast CT examination in preoperative period (a: axial, b: coranal). Collapsed germinative membrane (both long arrows) and free perihepatic fluid (short arrow).

\section{Case Report}

A 69-year-old woman was admitted to the Dicle University Hospital Emergency Department complaining of shortness of breath, abdominal pain, and vomiting. The patient had no history of trauma, prior surgery, or systemic disease. The physical examination revealed general abdominal tenderness. Her blood pressure, pulse, respiration rate, and temperature were $110 / 60 \mathrm{mmHg}, 103$ beats $/ \mathrm{min}, 20 / \mathrm{min}$, and $38.7^{\circ} \mathrm{C}$, respectively. The white blood cell count, hemoglobin, hematocrit, and platelet count were $14,300 / \mathrm{mm}^{3}, 11.9 \mathrm{mg} / \mathrm{dL}, 34.5 \mathrm{~g} / \mathrm{dL}$, and $332,000 /$ $\mathrm{mm}^{3}$, respectively. Other biochemical parameters, including the bilirubin level, were within the normal range. Nothing significant was observed in the posteroanterior chest $X$-ray or standing abdominal plain X-ray. Abdominal USG showed a $68-\times 57-\mathrm{mm}$ lesion with irregular borders and some cystic and echogenic areas in the left lobe of the liver. Minimal fluid was detected in the perihepatic region and lower abdomen. Abdominal CT revealed a collapsed germinative membrane $68 \times 92 \mathrm{~mm}$ in diameter and widespread intra-abdominal free fluid (Fig. 1). A laparotomy was performed, with a diagnosis of hydatid cyst rupture. At surgery, a $10-\times 8$-mm cyst with a tear on the inferior surface was observed. Seropurulent fluid and daughter vesicles were also disseminated throughout the abdomen. The other abdominal organs were normal grossly. After a partial cystectomy, the cyst pouch was irrigated with hypertonic saline (3\%), and the peritoneal cavity was washed with isotonic saline for 10 to 15 minutes. Then, two 22F Foley catheters were inserted: one into the cystic cavity and the other into the abdominal cavity. The patient was discharged without any trouble on the seventh postoperative day and prescribed $10 \mathrm{mg} / \mathrm{kg} / \mathrm{d}$ albendazole (Andazol, 
Biofarma, Istanbul, Turkey) for 3 months (3 weeks of drug administration and a 1-week drug-free interval), with liver enzyme and blood count monitoring. No recurrence or additional pathology was detected on CT at the 3-, 6-, and 9-month follow-ups.

\section{Discussion}

Although hydatid disease can be observed worldwide, it is endemic in Asia, Australia, the Middle East, Southern Europe, Africa, and South America. The liver is the organ most commonly affected by hydatid disease, yet E. granulosus can settle in practically any organ. ${ }^{4}$ In most cases, the disease remains silent clinically for years in the organ where it has settled. The symptoms are generally due to pressure resulting from growth of the cyst and to complications. ${ }^{5}$ The most common complication of a hepatic hydatid cyst is intrabiliary rupture $(5 \%-$ $25 \%){ }^{6}$ whereas intraperitoneal rupture is observed in $3.2 \%$ to $16 \%$ of the cases. ${ }^{2,6-9}$ Our patient's initial clinical findings were those of an acute abdomen.

Rupture can occur spontaneously or after trauma, depending on the diameter of the cyst $(\geq 10 \mathrm{~cm})$ or increased intracystic pressure. Our patient had no history of trauma before admission. This relatively rare $(1 \%-8 \%)$ condition occurs most often in younger patients, specifically those with superficially located and bigger lesions. ${ }^{1,10,11}$ Hydatid cysts that rupture intraperitoneally can present in various ways ranging from mild abdominal pain to potentially fatal anaphylactic reactions. ${ }^{4,12,13}$ The most common symptom on admission is abdominal pain, which is accompanied by vomiting and nausea in most patients. Dissemination of the hydatid fluid and protoscoleces into the peritoneal cavity can evoke allergy-related reactions, like urticaria, fever, hypotension, or anaphylaxis. ${ }^{1,11}$ Our patient presented with an acute abdomen.

The diagnosis of a ruptured hydatid cyst should be prompt because it requires emergency intervention. Because most cases are asymptomatic, the diagnosis is generally made after clinical or radiologic investigations for other reasons. ${ }^{14}$ USG and CT are useful for identifying intra-abdominal fluid and a cyst with a detached membrane. The sensitivities of these methods are $85 \%$ and $100 \%$, respectively. ${ }^{2,12}$

The preferred curative treatment for a perforated hydatid cyst is surgery. The methods used in the surgical treatment can be radical (pericystectomy + hepatic resection) or conservative (unroofing, with various procedures to treat the residual cavity). ${ }^{15}$ Radical surgery in the case of rupture may be difficult under emergency conditions. Conservative procedures are much easier, faster, and safer. ${ }^{7}$ All of the cyst contents should be removed, and the cyst and peritoneal cavities should be washed with saline solution and scolicidal agents. Hypertonic saline (3\%, $15 \%$, or $30 \%$ ) is scolocidal. Hypertonic saline may cause hypernatremia. ${ }^{16}$ In our patient, we used 3\% saline for the cyst pouch and isotonic saline for the peritoneal cavity. We did not encounter any significant complications using these solutions. All patients should be treated with albendazole for 2 to 3 months to decrease recurrences and followed with serologic and imaging tests for at least 6 months. Our patient was treated with albendazole $(10 \mathrm{mg} / \mathrm{kg} / \mathrm{d})$ for 3 months postoperatively.

In conclusion, spontaneous rupture of a hepatic hydatid cyst, albeit rare, may be fatal. Therefore, a ruptured hydatid cyst should be included in the differential diagnosis of acute abdomen, especially in regions where the disease is endemic. Given the high risk of anaphylactic reaction, a rapid diagnosis and intervention are mandatory. Surgery and postoperative medical treatment constitute the basis of treatment.

\section{Acknowledgments}

This article was presented as poster in the 8th National Congress of Trauma and Emergency Surgery, September 14-18, 2011, in Antalya, Turkey.

\section{References}

1. Derici H, Tansug T, Reyhan E, Dogan A, Nazli O. Acute intraperitoneal rupture of hydatid cysts. World J Surg 2006;30(10):E1879-E1883

2. Ray S, Das K. Spontaneous intraperitoneal rupture of hepatic hydatid cyst with biliary peritonitis: a case report. Cases J 2009;2:6511

3. Sumer A, Caglayan K, Altinli E, Koksal N. Case report: spontaneous liver hydatid cyst rupture in a child. Isr J Emerg Med 2009;9(1):13-16

4. Kok AN, Yurtman T, Aydin NE. Sudden death due to ruptured hydatid cysts of the liver. J Forensic Sci 1993;38(4):978-980

5. Karakaya K. Spontaneous rupture of a hepatic hydatıd cyst into the peritoneum causing only mild abdominal pain: a case report. World J Gastroenterol 2007;13(5): 806-808

6. Gulalp B, Koseoglu Z, Toprak N, Satar S, Sebe A, Gokel Y et al. Ruptured hydatid cyst following minimal trauma and few signs on presentation. Neth J Med 2007;65(6):117-118

7. Ozturk G, Aydinli B, Yildirgan MI, Basoglu M, Atamanalp SS, Polat KY et al. Posttraumatic free intraperitoneal rupture of liver cystic echinococcosis: a case series and review of literature. Am J Surg 2007;194(3):313-316 
8. Prousalidis J, Tzardinoglou K, Sgouradis L, Katsohis C, Aletras H. Uncommon sites of hydatid disease. World J Surg 1998;22(1):17-22

9. Karavias DD, Vagianos CE, Kakkos SK, Panagopoulos CM, Androulakis JA. Peritoneal echinococcosis. World J Surg 1996;20(3):337-340

10. Yuksel M, Kir A, Ercan S, Batirel HF, Baysungur V. Correlation between sizes and intracystic pressures of hydatid cysts. Eur J Cardiothorac Surg 1997;12(6):903-906

11. Patel SS, Butt AA. Inadvertent rupture of an echinococcal cyst: case report and review of literature. Am J Med Sci 2004; 327(5):268-271

12. Akcan A, Akyildiz H, Artis T, Ozturk A, Deneme MA, Ok E et al. Peritoneal perforation of liver hydatid cysts: clinical presentation, predisposing factors, and surgical outcome. World J Surg 2007;31(6):1284-1291

13. Dirican A, Unal B, Ozgor D, Piskin T, Aydin C, Sumer F et al. Perforated hepatic hydatid cyst into the peritoneum with mild symptoms. Case Rep Gastroenterol 2008;2(3):439-443

14. Lo Casto A, Salerno S, Grisanti M, Mastrandrea G. Hydatid cyst of the liver communicating with the left colon. Br J Radiol 1997;70(834):650-651

15. Dziri C, Haouet K, Fingerhut A. Treatment of hydatid cyst of the liver: where is the evidence? World J Surg 2004;28(8): 731-736

16. Akcan A, Sozuer E, Akyildiz H, Ozturk A, Atalay, Yilmaz Z. Predisposing factors and surgical outcome of complicated liver hydatid cysts. World J Gastroenterol 2010;28(24):3040-3048 